\title{
RELIABILITY ANALYSIS OF A CRASHED THIN-WALLED S-RAIL ACCOUNTING FOR RANDOM SPOT WELD FAILURES
}

\author{
R. Stocki ${ }^{1}$, P. Tauzowski, J. Knabel \\ Institute of Fundamental Technological Research, Polish Academy of Sciences, \\ Swietokrzyska 21, 00-049 Warsaw, Poland
}

\begin{abstract}
Possibly the most common application of spot welding is in the automobile manufacturing industry, where it is almost universally used to weld the sheet metal car components. However, due to manufacturing inaccuracies and fatigue failures an important number of spot welds may be missing in an operational vehicle. It seems that in order to properly analyze reliability of such structures, in particular crashworthiness reliability, the spot weld failures must be considered. Representing properties of each spot weld in a stochastic model by corresponding random variables is extremely inefficient. Therefore, in the current paper an approach is proposed for handling spot weld defects in the reliability analysis by accounting for their averaged influence on a failure criterion. The approach consists in appropriate treatment of a random noise component of the limit state function. The noise results from strategy of deleting a certain number of randomly selected spot weld elements from the finite element model each time the limit state function value is computed.

Dealing with noisy limit state functions in structural reliability analysis is a challenging task. The only method that seems to be insensitive to this phenomenon is Monte Carlo sampling, which for most of the applications of practical interest is prohibitively expensive. Having this in mind, in the paper a method based on the algorithm proposed by Zou et al. in [23] is investigated. The method combines the best features of the first order reliability method, the response surface technique and the importance sampling method to achieve both accuracy and efficiency. A detailed study on the reliability of thin-walled s-rail subjected to crash is performed. Some suggestions concerning modification of the original algorithm are proposed.
\end{abstract}

Keywords: crashworthiness reliability, response surface approximation, adaptive importance sampling, spot weld failures

\footnotetext{
${ }^{1}$ Corresponding author, email: rstocki@ippt.gov.pl
} 


\section{Introduction}

In the reliability analysis of complex engineering systems, the limit state function (LSF) is generally implicit and very often, highly nonlinear. Furthermore, each function evaluation is usually computationally expensive. This is the case of virtual crash simulation. Even with present-day parallel machines the time to obtain a structural response is very long and only a limited number of such computer experiments can be afforded. It is therefore extremely important to choose a reliability analysis method that minimizes the number of function evaluations needed for an estimation of the failure probability $P_{f}$, which is of an acceptable accuracy.

Nonlinearity of the limit state functions in crash related problems arises not only from the physical nature of the modelled phenomenon but also from the numerical noise introduced by a computer crash simulation method, e.g., explicit integration of the dynamic equilibrium problem. Such a numerical noise (high sensitivity to parameter variations) greatly impairs a direct application of efficient gradient-based reliability analysis methods. However, this noise is different from the one observed in physical experiments, where repeated identical tests usually lead to a scatter of measured results. Contrary to laboratory tests, repeated runs of a computational code for the same set of input parameters on the same hardware platform result with the same values of structural performances. On the other hand, there are problems where it is computationally advantageous to deal with this type of random noise in reliability analysis rather than to precisely model its sources with random variables and/or processes. Crashworthiness reliability analysis accounting for random failures of spot weld connections in vehicle components seems to be such a case. It has been observed that due to manufacturing inaccuracies, fatigue failures and minor accidents a substantial number of spot welds may be weakened or even absent in an operational vehicle, see [3]. Associating random variables with material properties of each finite element used to represent a spot weld is highly inefficient, let aside availability of the relevant statistical data. Therefore, to represent somehow spot weld failures in reliability computations and to make this task manageable for practical problems a possible approach is to delete certain number of randomly selected spot weld elements each time the LSF value is computed, producing a noise like effect. This technique, however, requires a reliability analysis method that is able to cope with the noise. A study on such a method with a special emphasis on crashworthiness reliability problems is the main subject of the current paper.

Even though stochastic analysis for crashworthiness related problems and in particular crash- 
worthiness reliability seem to nowadays gain more and more interest the number of papers proposing reliability analysis methods for computer simulated crash problems is still low. Most of the publications available for the authors address these problems only in the context of reliability based design optimization (RBDO) of automotive structures, see, e.g., [5, 9, 21]. These papers primarily emphasize improvements of optimization algorithms. In order to reduce huge computational cost of RBDO the basic reliability analysis methods, providing only a rough failure probability estimation, are usually selected. Unfortunately, despite of the very strong sensitivity of the optimal design to design parameter variations, optimization for crashworthiness is still often considered as deterministic optimization problem, see, e.g., [6, 16, 18, 20, 22]. Most of the crashworthiness reliability analysis methods reported so far are based to some extent on the LSF approximation by means of a response surface. The response surface methodology is a valuable tool for problems with implicit limit state functions. However, for problems with large number of random variables, even efficient design of experiments (DOE) techniques such as fractional factorials, central composite design or Box-Behnken design become unaffordable. When using a response surface as a surrogate of the actual LSF the accuracy of reliability estimate is greatly affected by the accuracy of the response surface approximation. Therefore, it is rather difficult to propose an accurate and efficient reliability estimation by using the traditional response surface approach.

Having in mind application to crashworthiness reliability problems and the particular treatment of spot weld failures in the current paper a reliability analysis method based on the algorithm proposed by Zou, Mahadevan, Mourelatos, and Meernik [23, 24] and called here as ZMMM, is investigated. The two phase algorithm consists of the search of the most probable failure point using the adaptive response surface approach and eventually the multimodal adaptive importance sampling. It combines the best features of the first order reliability method (FORM), the response surface methodology and importance sampling to achieve both accuracy and efficiency. The detailed presentation of the method, which closely follows the presentation in the original article [23], together with some modifications and critical comments are presented in Sec. 3. In Sec. 4 is presented a study on reliability of the thin-walled s-rail subjected to crash. Some suggestions concerning modifications of the ZMMM algorithm are also proposed. 


\section{Reliability analysis problem formulation}

Stochastic parameters describing a structural system are usually modelled as random variables, $X_{1}, X_{2}, \ldots, X_{n}$. They are called the basic variables and constitute a random vector $\boldsymbol{X}$ whose samples $\boldsymbol{x}=\left[x_{1}, x_{2}, \ldots, x_{n}\right]^{\mathrm{T}}$ belong to the Euclidian space. In the space $\mathcal{X}$ the probability measure is defined by the joint probability density function (PDF) $f_{\boldsymbol{X}}(\boldsymbol{x})$ of the random vector $\boldsymbol{X}$. Depending on the sample values of the basic variables, the system will satisfy design requirements or not (fail). The criterion of structural failure is usually expressed by the equality $g(\boldsymbol{x})=0$ that defines a hypersurface, called the limit state surface, in the space $\mathcal{X}$. It divides the space $\mathcal{X}$ into two regions: the failure domain $\Omega_{f}=\{\boldsymbol{x}: g(\boldsymbol{x}) \leq 0\}$ and the safe domain $\Omega_{s}=\{\boldsymbol{x}: g(\boldsymbol{x})>0\}$. Hence, the failure probability of the structural system is determined by the following integral:

$$
P_{f}=\mathbb{P}\left[\boldsymbol{X} \in \Omega_{f}\right]=\mathbb{P}[g(\boldsymbol{X}) \leq 0]=\int_{\Omega_{f}} f_{\boldsymbol{X}}(\boldsymbol{x}) \mathrm{d} \boldsymbol{x}
$$

where $\mathbb{P}[A]$ means the probability of the random event $A$. In applications, where the number $n$ of basic variables $X_{i}$ can be great, the integral domain $\Omega_{f}$ complex and the calculation of the limit state function $g(x)$ cumbersome, e.g. involving a finite element numerical procedure, the direct integration appears to be impractical. Therefore, some approximate methods have been developed that allow in many cases for effective reliability assessment of structural systems. In the approach that is most commonly used in application, the problem of the reliability calculation is appropriately transformed, $\boldsymbol{U}=\boldsymbol{T}(\boldsymbol{X})$ (see e.g. [12, 2, 15]), into the space $\mathcal{U}$ where the probability measure is defined by the probability density function $f_{\boldsymbol{U}}(\boldsymbol{u})=\prod_{i=1}^{n} \varphi\left(u_{i}\right)$ being the product of the $n$ one-dimensional standard normal PDFs of random variables $U_{i}=T_{i}(\boldsymbol{X})$. Since the limit state condition is also transformed into $\mathcal{U}, g(\boldsymbol{x})=0 \rightarrow h(\boldsymbol{u})=g\left[\boldsymbol{T}^{-1}(\boldsymbol{u})\right]=0$, the failure probability can be calculated as follows:

$$
P_{f}=\mathbb{P}[h(\boldsymbol{U}) \leq 0]=\int_{\{\boldsymbol{u}: h(\boldsymbol{u}) \leq 0\}} f_{\boldsymbol{U}}(\boldsymbol{u}) \mathrm{d} \boldsymbol{u}=\int_{\Delta_{f}} f_{\boldsymbol{U}}(\boldsymbol{u}) \mathrm{d} \boldsymbol{u},
$$

where $\Delta_{f}$ denotes the failure domain $\Omega_{f}$ transformed to the $\mathcal{U}$ space.

The axial symmetry of $f_{\boldsymbol{U}}(\boldsymbol{u})$ assures for any linear function $l(\boldsymbol{u})=\beta-\boldsymbol{\alpha}^{\mathrm{T}} \boldsymbol{u}=0$, the following equality to be true

$$
\mathbb{P}[l(\boldsymbol{U}) \leq 0]=\int_{\{\boldsymbol{u}: l(\boldsymbol{u}) \leq 0\}} f_{\boldsymbol{U}}(\boldsymbol{u}) \mathrm{d} \boldsymbol{u}=\Phi(-\beta),
$$


where the coefficients, $-\alpha_{i}, i=1,2, \ldots, n$, are the components of the normalized gradient of the hyperplane $l(\boldsymbol{u})=0$, i.e. $\boldsymbol{\alpha}^{\mathrm{T}} \boldsymbol{\alpha}=1, \beta=\operatorname{sign}[l(\mathbf{0})] \delta$ is the signed distance $\delta$ between the hyperplane and the origin in $\mathcal{U}$ and $\Phi(\cdot)$ is the standard normal distribution. Thus, the linear approximation of the transformed limit state surface $h(\boldsymbol{u})=0$ in the point closest to the origin provides a simple estimate of the failure probability of structural system

$$
P_{f}=\mathbb{P}[h(\boldsymbol{U}) \leq 0] \approx \mathbb{P}[l(\boldsymbol{U}) \leq 0]=\Phi(-\beta),
$$

where $\beta$ is called the first order reliability index. The approach based on the linear approximation of the transformed limit state surface is called the first order reliability method (FORM). The reliability index is determined as a solution of the following optimization problem:

$$
\begin{gathered}
\beta=\operatorname{sign}[h(\mathbf{0})] \delta^{*} \\
\delta^{*} \equiv\left\|\boldsymbol{u}^{*}\right\|=\min \|\boldsymbol{u}\| \quad \text { subject to: } h(\boldsymbol{u})=0 .
\end{gathered}
$$

The point $\boldsymbol{u}^{*}$ is usually referred in the literature as design point or the Most Probable failure Point (MPP). The problem (5) can, in theory, be solved by any nonlinear optimization algorithm (cf. [11] for comparison of various methods), however, two algorithms, namely the RackwitzFiessler algorithm [1] and NLPQL [17] are commonly assumed to be the most efficient ones. Unfortunately, such an observation is only true for smooth and differentiable LSFs which is not the case of reliability problems considered in this paper. Therefore, a method that is tailored for specific needs of crashworthiness reliability has to be devised.

A viable alternative to FORM approach are simulation methods. This is mainly due to the fast development of computational facilities. Still, for systems with a low failure probability a large number of simulations will have to be performed to get a stable estimate of the final result. Hence, in practise some variance reduction techniques must be used, e.g. importance sampling, where sampling is performed in the region where failures are more likely to occur (see, e.g., [15] for the review of simulation methods in reliability analysis).

To present the idea of simulation techniques it is convenient to introduce the indicator function of the failure domain, defined as follows:

$$
I_{\Omega_{f}}(\boldsymbol{x})=\left\{\begin{array}{ll}
1 & \text { if } \boldsymbol{x} \in \Omega_{f} \\
0 & \text { if } \boldsymbol{x} \notin \Omega_{f}
\end{array} .\right.
$$

In the crude Monte Carlo approach the samples $\boldsymbol{x}$ of the random vector $\boldsymbol{X}$ are being generated using the joint PDF $f_{\boldsymbol{X}}(\boldsymbol{x})$ and the following estimator is employed for the probability of failure 
computation

$$
\widehat{P}_{f}=\frac{1}{K} \sum_{i=1}^{K} I_{\Omega_{f}}\left(\boldsymbol{X}_{i}\right),
$$

where $\boldsymbol{X}_{i}$ are the independent random vectors with the joint PDF $f_{\boldsymbol{X}}(\boldsymbol{x})$ and $K$ is the number of sample points. By computing the mean value and the standard deviation of the above estimator it can be shown that its coefficient of variation is given by

$$
\nu_{\widehat{P}_{f}}=\frac{\sigma_{\widehat{P}_{f}}}{\mathbb{E}\left[\widehat{P}_{f}\right]}=\sqrt{\frac{1-P_{f}}{K P_{f}}} .
$$

It is easy to check that for problems where the expected probabilities of failure are low, $10^{-7} \div$ $10^{-3}$, to get an accurate result, say $\nu_{\widehat{P}_{f}}=5 \%$, it is required to perform $K=4 \cdot 10^{5} \div 4 \cdot 10^{9}$ simulations. This computational burden is certainly not acceptable, especially when obtaining LSF values requires a nonlinear finite element analysis.

The method that allows to significantly reduce the number of required simulations is the importance sampling. It can be shown that in the $\mathcal{U}$ space the importance sampling estimator for failure probability $\widehat{P}_{f}$ takes the form

$$
\widehat{P_{f}}=\frac{1}{K} \sum_{i=1}^{K} I_{\Delta_{f}}\left(\boldsymbol{V}_{i}\right) \frac{\varphi_{n}\left(\boldsymbol{V}_{i}, \mathbf{0}, \boldsymbol{I}\right)}{s_{\boldsymbol{V}}\left(\boldsymbol{V}_{i}\right)},
$$

where $s_{\boldsymbol{V}}(\cdot)$ is the joint PDF (also called the sampling density) of the $n$-element random vector $\boldsymbol{V}, I_{\Delta_{f}}$ is the indicator function of the failure domain $\Delta_{f}$ in the $\mathcal{U}$ space and $\varphi_{n}(\cdot, \mathbf{0}, \boldsymbol{I})$ is the $n$-dimensional normal joint PDF with zero mean values and unit covariance matrix. In order to compute the value of the above estimator we use the realizations $\boldsymbol{v}_{i}$ of the vector $\boldsymbol{V}_{i}$ generated from $s_{\boldsymbol{V}}(\cdot)$. The key element for the efficiency of importance sampling method is a good choice of the sampling density that should minimize the variance of (9). Unfortunately, in general, derivation of the optimal $s_{\boldsymbol{V}}(\cdot)$ function is difficult. However, very often quite substantial improvement of the computational efficiency can be obtained by selecting it as the $n$-dimensional normal PDF 'located' over the design point $\boldsymbol{u}^{*}$ - the region with the largest contribution to the value of $P_{f}$ (see [19])

$$
s_{\boldsymbol{V}}(\boldsymbol{v})=\varphi_{n}\left(\boldsymbol{v}, \boldsymbol{u}^{*}, \boldsymbol{I}\right)=\prod_{i=1}^{n} \varphi\left(v_{i}-u_{i}^{*}\right) .
$$

In such a case the estimator is given by

$$
\widehat{P_{f}}=\frac{1}{K} \sum_{i=1}^{K} I_{\Delta_{f}}\left(\boldsymbol{V}_{i}\right) \frac{\varphi_{n}\left(\boldsymbol{V}_{i}, \mathbf{0}, \boldsymbol{I}\right)}{\varphi_{n}\left(\boldsymbol{V}_{i}, \boldsymbol{u}^{*}, \boldsymbol{I}\right)} .
$$


With the choice of multi-normal sampling density (10) the estimate of $P_{f}$ is not very sensitive to the shape of $\Delta_{f}$ and unless it is extremely nonlinear, the "success" rate for sample points selected from $s_{\boldsymbol{V}}(\cdot)$ is about $50 \%$ (i.e. approximately equal likelihood of falling into either the safe or the failure domain). This is in marked contrast to the conventional Monte Carlo method for which the probability of having a sample point in the failure region is approximately equal to the probability of failure to be computed. Experience shows, that in most of the cases only few thousand simulations are necessary to get good $P_{f}$ assessment with the estimator (11) (the estimation quality measured by the coefficient of variation of the estimator), many orders of magnitude less than for crude Monte Carlo sampling.

Of course, there exist some limitations to the above importance sampling procedure (listing after [14] only these relevant to sampling in the $\mathcal{U}$ space and single LSF).

1. The sampling distribution $s_{\boldsymbol{V}}(\cdot)$ may not be well chosen.

2. The LSF under consideration have many design points (local minima of the function $\|\boldsymbol{u}\|$ provided $h(\boldsymbol{u})=0$ ).

3. Extremely concave LSFs which lead to low efficiency in sampling.

A potential remedy for these problems offer adaptive methods of selecting the sampling density. It can be shown that the importance sampling is more effective when the sampling PDF $s_{\boldsymbol{V}}(\cdot)$ is more closely proportional to the original sampling density in the failure region $\Omega_{f}$ (or $\Delta_{f}$ if sampling in the $\mathcal{U}$ space). A popular approach is to let $s_{\boldsymbol{V}}(\cdot)$ be a composite of $k$ pre-selected elementary sampling densities $s_{\boldsymbol{V}}^{(i)}(\cdot)$

$$
s_{\boldsymbol{V}}(\boldsymbol{v})=\sum_{i=1}^{k} w^{(i)} s_{\boldsymbol{V}}^{(i)}(\boldsymbol{v}),
$$

where $w^{(i)}, i=1, \ldots, k$ are weights to be determined so as to let $s_{\boldsymbol{V}}(\cdot)$ approach $f_{\boldsymbol{X}}(\cdot)$ in $\Omega_{f}$ (or $\varphi_{n}(\cdot, \mathbf{0}, \boldsymbol{I})$ in $\Delta_{f}$ ). Each of the $s_{\boldsymbol{V}}^{(i)}(\cdot)$ in Eq. (12) may be pre-selected or may be updated with increased knowledge of the problem as sampling progresses. In [8] Karamchandani et al. selected $s_{\boldsymbol{V}}^{(i)}(\cdot), i=1, \ldots, k$ to be equal to the original PDF but with mean shifted to some selected representative points, denoted $\hat{\boldsymbol{v}}^{(i)}$. The corresponding weights are defined (in case of 
sampling in the standard normal space) as

$$
w^{(i)}=\frac{\varphi_{n}\left(\hat{\boldsymbol{v}}^{(i)}, \mathbf{0}, \boldsymbol{I}\right)}{\sum_{j=1}^{k} \varphi_{n}\left(\hat{\boldsymbol{v}}^{(j)}, \mathbf{0}, \boldsymbol{I}\right)} .
$$

This means that the weights are proportional to the contributions of the respective representative point to the current estimate of the failure probability. It should be clear that the effectiveness of sampling in developing the ideal sampling density depends very much on the set of initial $k$ representative points and on the initial sampling distribution from which they are drown.

A slightly modified version of the above adaptive importance sampling method, is presented in section 3.2, where the algorithm of choosing the representative points and updating the sampling density is described in detail.

\section{Description of the chosen reliability analysis method}

The proposed approach is composed of two major parts. In the first part, the MPP search is performed by means of adaptive response surface based on the so-called optimal Latin hypercube (OLH) design of experiments, see, e.g., [10]. In the second part, developing further the idea of Karamchandani et al. [8] a multi-modal adaptive importance sampling method is proposed to improve the estimate of the first part. All the useful information from the first part, especially concerning the MPP location, is passed to the second stage to ensure efficiency of the adaptive importance sampling technique.

\subsection{First part: Most probable failure point search}

The main issue in the presented algorithm is to enable its convergence for highly nonlinear limit states. The most probable failure point is obtained by repeatedly searching for the local MPP (by some constrained optimization procedure) within the current "trust region" and updating the trust region until convergence. A polynomial response surface is used for the local approximation of the implicit LSF. It is based on sample points generated within the trust region by the OLH design.

The following steps describe the iterative procedure realized in the standard normal space: 
1. Define the initial trust region (box) - its position and size.

Usually, the initial box center is assumed at the origin and the size has to be big enough to include the sample points from both the safe and failure domains. This, of course, implies some initial knowledge of the problem. If no prior information is given the hypercube with the side length equal to 6 may be assumed, which corresponds to $\left[-3 \sigma_{X_{i}}, 3 \sigma_{X_{i}}\right]$ ranges of random variables, where $\sigma_{X_{i}}$ is the standard deviation of the $i$-th random variable.

\section{Generate a number of points using the OLH-based DOE.}

The sample points are realizations of uniformly distributed random variables with mean values at the box center and the bounds determined by the box size. The points are transformed back to the original space and the corresponding LSF values are computed. Another DOEs, like central composite design, factorial design or axial design can be also used provided that the number of variables is not to large.

\section{Build a local LSF approximation with linear or quadratic response surface function.}

In the study presented in this article the moving least-squares approach (MLS) is used for finding coefficients of the regression equation. For the MLS method, weights are assigned to the squared difference between OLH sample points and a point $\boldsymbol{u}$ where the LSF approximation is to be computed. By using a weight function $w\left(\boldsymbol{u}-\boldsymbol{u}_{i}\right)$, more emphasis/weight is locally placed on those experiments $\boldsymbol{u}_{i}$, which are close to $\boldsymbol{u}$. Hence, the response surface coefficients are reevaluated each time a new $\boldsymbol{u}$ point is considered. Such models are able to account for higher than second-order nonlinearity with simple polynomial models. The LSF model $\tilde{h}(\boldsymbol{u})$ built upon the results of designed experiments has the form

$$
\tilde{h}(\boldsymbol{u})=\boldsymbol{a}^{\mathrm{T}}(\boldsymbol{u}) \boldsymbol{b}(\boldsymbol{u})
$$

where

$$
\widehat{\boldsymbol{b}}=\left(\boldsymbol{A}^{\mathrm{T}} \boldsymbol{W} \boldsymbol{A}\right)^{-1} \boldsymbol{A}^{\mathrm{T}} \boldsymbol{W} \boldsymbol{h}
$$

is the least-square estimate for the unknown parameters $\boldsymbol{b}, \boldsymbol{W}$ is the weights matrix, $\boldsymbol{A}$ the regression design matrix, $\boldsymbol{a}$ the vector of the linear independent regression functions and $\boldsymbol{h}$ is the vector of LSF values computed at OLH generated experimental points. An exponential, multi-dimensional weight function similar to Gaussian distribution is usually 
employed. $\boldsymbol{W}$ is a diagonal matrix with the weights

$$
w_{i i}=\exp \left[-\sum_{j=1}^{n}\left(u_{i j}^{\mathrm{ex}}-u_{j}\right)^{2} /\left(2 n \eta^{2}\right)\right], \quad \eta>0 .
$$

In the above equation $u_{i j}^{\mathrm{ex}}$ stands for the $j$-th component of the $i$-th experimental point, $u_{j}$ is the $j$-th component of the reference point $\boldsymbol{u}$ and $\eta$ is a parameter controlling the shape of the weight function.

4. Find an approximate MPP location by solving the following optimization problem:

$$
\begin{aligned}
\text { find: } & \boldsymbol{u}, \\
\text { that minimizes: } & \|\boldsymbol{u}\|^{2}=\boldsymbol{u}^{\mathrm{T}} \boldsymbol{u}, \\
\text { subject to: } & \tilde{h}(\boldsymbol{u})=0,
\end{aligned}
$$

where $\tilde{h}(\boldsymbol{u})$ is the local approximation of the LSF given by Eq. (14). The exact LSF value corresponding to MPP is computed and the point is added to the database of generated experimental points.

\section{Move the trust region center and decide if to change its size}

5.1 If the MPP found in the previous step is inside the trust region then the box center is moved there and the size of the trust region (measured by the length of the hypercube's side) is reduced. The reduction strategy is a very important factor for the convergence of the algorithm. The solution proposed in [23], consists in dividing the side of the box by a constant factor e.g., by two. Unfortunately, such an approach can lead to too rapid trust region reduction, which may greatly impair the convergence, especially for problems with many random variables and noisy LSFs. The method adopted in this study consists in dividing the volume of the trust region rather than dividing the side length. This reduction strategy is discussed in more detail in Sec. 4.

5.2 If the MPP found as the solution of the problem (17)-(19) is outside the trust region then it is projected on the trust region boundary in the direction of the current trust region center. The projection point becomes the new box center but the trust region size is not changed. However, sometimes it may be advantageous to enlarge it, especially when a number of consecutive iterations lead to MPPs outside the trust 
region. This is usually the case for nonlinear and/or noisy LSFs. Basing on the experience gained by performing a number of numerical tests it is proposed to adopt a strategy where three consecutive MPPs outside the trust region result in the trust region expansion by the same factor as used for the reduction.

\section{Repeat the steps 2 to 5 until the convergence criteria are satisfied.}

The convergence check is performed only if MPP is found inside the trust region (see step 5.1). In addition to the stop criterion based on the distance between the last two iteration points (box centers), it is proposed to verify if the MPP approximation is really located on the limit state surface $h(\boldsymbol{u})=0$. In order to account for the LSF noise multiple LSF computations at MPP are performed and the mean LSF value is checked whether it is in an epsilon vicinity of zero or not.

When constructing a local LSF approximation, all the points that 'fall' into the current box should be used in the regression analysis, i.e. not only newly generated points but also the sample points from previous iterations.

\subsection{Second part: Multi-modal adaptive importance sampling}

As it has been already mentioned, the second part of the method is the modified Karamchandani procedure of the multi-modal adaptive importance sampling. It is described below:

1. Generate $m_{0}$ sample points using the original joint PDF of the random vector $\boldsymbol{U}$ but with the means shifted to MPP. Compute the corresponding LSF values.

In [24] it is suggested to take $m_{0}=10$ to 50 , however, it seems more appropriate to relate $m_{0}$ to the space dimension $n$, e.g., $m_{0}=5 n \div 10 n$. In the example analyzed in Sec. $4 m_{0}=10 n$ points are used. Keeping the convention adopted in section 2 of denoting by $\boldsymbol{V}$ the "sampling variables" and remembering that sampling is performed in the standard normal space, the PDF used to generate $m_{0}$ sample points is $\varphi_{n}\left(\boldsymbol{v}, \boldsymbol{u}^{*}, \boldsymbol{I}\right)$, where $\boldsymbol{u}^{*}=\hat{\boldsymbol{v}}^{(1)}$ is the MPP.

2. Determine $k$ representative points from all the points generated so far.

By all the points we mean points generated during the second as well as the first part of the algorithm. The current representative status of some points is disregarded. The procedure is schematically shown in Fig. 1 and can be described as follows: 


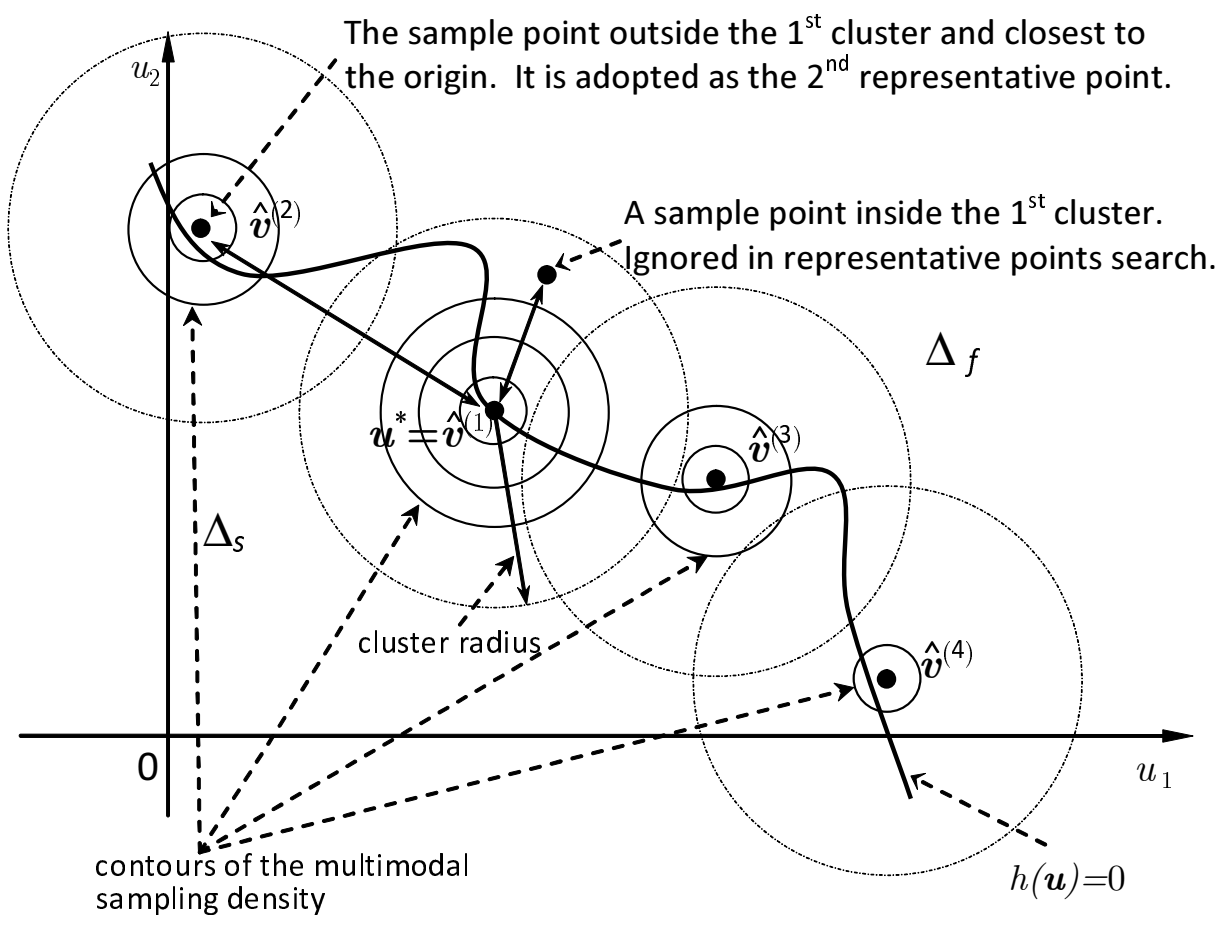

Figure 1: General idea of multi-modal adaptive importance sampling strategy.

2.1 Select a sample point with the highest original PDF in the failure domain.

2.2 Eliminate all other points within a specified cluster radius around the selected point. The cluster radius may be set equal to half of the distance from MPP to the origin $(\beta / 2)$, say.

2.3 Find the point with the highest probability density among the remaining points, excluding those that have been already selected as representative points.

2.4 Repeat the sub-steps 2.2 and 2.3 until no points are left.

3. Calculate the coefficient of variation of the failure probability estimator $\nu_{\widehat{P}_{f}}$ corresponding to $m_{0}$ sample points.

It is given by

where

$$
\nu_{\widehat{P}_{f}}=\frac{\sqrt{\mathbb{V a r}\left[\widehat{P}_{f}\right]}}{\widehat{P}_{f}}
$$

$$
\widehat{P}_{f}=\frac{1}{m_{0}} \sum_{i=1}^{m_{0}} I_{\Delta_{f}}\left(\boldsymbol{v}_{i}\right) \frac{\varphi_{n}\left(\boldsymbol{v}_{i}, \mathbf{0}, \boldsymbol{I}\right)}{s_{\boldsymbol{V}}\left(\boldsymbol{v}_{i}\right)},
$$




$$
\operatorname{Var}\left[\widehat{P}_{f}\right]=\frac{1}{m_{0}\left(m_{0}-1\right)} \sum_{i=1}^{m_{0}}\left(I_{\Delta_{f}}\left(\boldsymbol{v}_{i}\right) \frac{\varphi_{n}\left(\boldsymbol{v}_{i}, \mathbf{0}, \boldsymbol{I}\right)}{s_{\boldsymbol{V}}\left(\boldsymbol{v}_{i}\right)}-\widehat{P}_{f}\right)^{2},
$$

and $s_{\boldsymbol{V}}(\boldsymbol{v})$ is the multi-modal PDF defined by Eq. (24) and $I_{\Delta_{f}}(\boldsymbol{v})$ is the failure domain indicator function. For the first sample of $m_{0}$ points $s_{\boldsymbol{V}}(\boldsymbol{v})=\varphi_{n}\left(\boldsymbol{v}, \hat{\boldsymbol{v}}^{(1)}, \boldsymbol{I}\right)$. If $\nu_{\widehat{P}_{f}}$ is greater than some target value, the procedure is continued, otherwise, go to step 5.

4. Use the current $k$ representative points to construct a multi-modal sampling density $s_{\boldsymbol{V}}(\boldsymbol{v})$ as follows:

4.1 Calculate the value of the original $\operatorname{PDF} \varphi_{n}(\boldsymbol{v}, \mathbf{0}, \boldsymbol{I})$ for each representative point.

4.2 Calculate the weight for each representative point $\hat{\boldsymbol{v}}^{(j)}, j=1, \ldots, k$, as (see Eq. (13))

$$
\hat{w}^{(j)}=\frac{\varphi_{n}\left(\hat{\boldsymbol{v}}^{(j)}, \mathbf{0}, \boldsymbol{I}\right)}{\sum_{r=1}^{k} \varphi_{n}\left(\hat{\boldsymbol{v}}^{(r)}, \mathbf{0}, \boldsymbol{I}\right)} .
$$

4.3 The multi-modal PDF $s_{\boldsymbol{V}}(\boldsymbol{v})$ is assumed to be the weighted sum of the probability densities corresponding to representative points, see Eq. (12)

$$
s_{\boldsymbol{V}}(\boldsymbol{v})=\sum_{j=1}^{k} \hat{w}^{(j)} \varphi_{n}\left(\boldsymbol{v}, \hat{\boldsymbol{v}}^{(j)}, \boldsymbol{I}\right),
$$

where $\varphi_{n}\left(\boldsymbol{v}, \hat{\boldsymbol{v}}^{(j)}, \boldsymbol{I}\right)$ denotes the original PDF with mean shifted to the $j$-th representative point $\hat{\boldsymbol{v}}^{(j)}$.

4.4 Generate $m_{1}$ additional points using $s_{\boldsymbol{V}}(\boldsymbol{v})$ from Eq. (24). It is suggested that $m_{1}$ is a multiple of the number of representative points, e.g., $m_{1}=5 k \div 10 k$. Compute the corresponding LSF values.

4.5 Update the value of $\widehat{P}_{f}$ then compute the updated value of $\mathbb{V} \operatorname{ar}\left[\widehat{P}_{f}\right]$ and finally coefficient of variation of the estimator $\nu_{\widehat{P}_{f}}$ using Eq. (20). Here, it must be remembered to replace $m_{0}$ by a total number of points generated in the sampling phase and to account for proper sampling densities when performing summations in (21) and (22). If $\nu_{\widehat{P}_{f}}$ is greater than some target value then the new representative points are determined according to the procedure described in step 2 and all the actions from the step 4 are repeated. If $\nu_{\widehat{P}_{f}}$ is smaller than the target value then the algorithm proceeds to step 5. In [23] it is suggested that the target value for $\nu_{\widehat{P}_{f}}$ should be taken in the range $0.2 \div 0.3$. 
5. Generate $m_{2}$ sample points using the multi-modal sampling density (24) constructed using the final set of representative points.

6. Calculate the probability of failure and the coefficient of variation using the formulas (20)-(22) accounting for the comments from the point 44.5 .

7. Repeat steps 5 and 6 until the value of $\widehat{P}_{f}$ converges

$$
\frac{\left|\widehat{P}_{f}^{(j+1)}-\widehat{P}_{f}^{(j)}\right|}{\widehat{P}_{f}^{(j)}} \leqslant \varepsilon,
$$

where $\varepsilon$ is the tolerance (e.g., $\varepsilon=0.1$ can be assumed) and $\widehat{P}_{f}^{(j)}$ is the probability of failure for the $j$-th iteration. The calculated coefficient $\nu_{\widehat{P}_{f}}$ should remain under the preset target value as well.

We can list several aspects of the presented approach that make it particularly attractive in the context of reliability analysis of crash related problems. They are as follows:

- The first part of the algorithm, the MPP search based on the LSF approximation by a response surface is relatively insensitive to the numerical noise inherent to crash simulations.

- No sample points are 'wasted'. The information generated in the first part is subsequently utilized for finding representative points in adaptive importance sampling part.

- The accuracy of the algorithm is easy to control. In case when only some crude estimate of the probability of failure is needed (or if it is all the budget permits) the importance sampling part can be skipped.

- The method has "potential" to deal with multiple MPPs.

\section{Reliability of s-rail subjected to crash}

Here we consider a thin-walled steel s-rail, shown in Fig. 2, clamped at one end and hit at the other end by the $100 \mathrm{~kg}$ mass moving with the initial velocity $v_{0}=15 \mathrm{~m} / \mathrm{s}(54 \mathrm{~km} / \mathrm{h})$ in the $x$-axis direction. The beam consists of 3 omega-shaped parts and the cover plate. The omega parts are attached to the cover with 64 spotwelds. The elastic-plastic-brittle material is assumed 
[7]. The finite element model consists of 5760 MITC4 type shell elements [4] and 64 spring elements to model the spotwelds. The finite element analysis is performed using Radioss [13], explicit finite element solver, developed for analysis of highly dynamic and nonlinear problems, in particular crash.

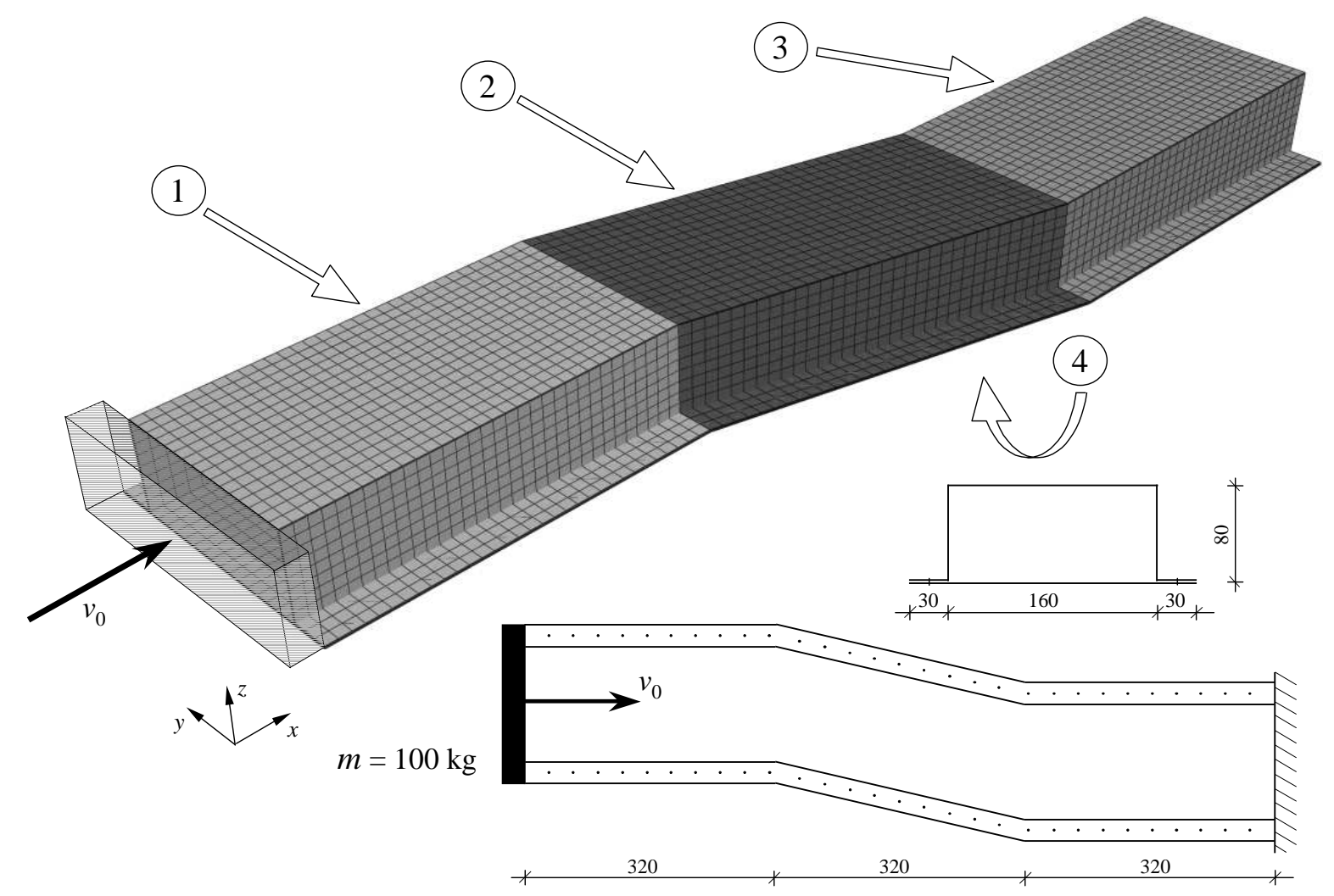

Figure 2: S-rail crash problem. The finite element model and geometry. Dimensions are in millimeters. The arrows indicate position of the parts.

The beam acts here as an energy absorbing device, so the major concern in its design is to ensure a good energy management by collapsing in regular folding rather than buckling mode (see Fig. 3). However, very often a design that performs satisfactorily in the ideal (nominal) operating conditions is not reliable due to large sensitivity to unavoidable uncertainties of some parameters. In reality it is hard to guarantee that the mass will impact the beam precisely in the assumed direction that all the spotwelds are well manufactured and the thicknesses of metal parts do not differ from their nominal values. For this reasons it seems essential that a design is verified for its sensitivity to parameter uncertainties and, if possible, to assess the probability of 

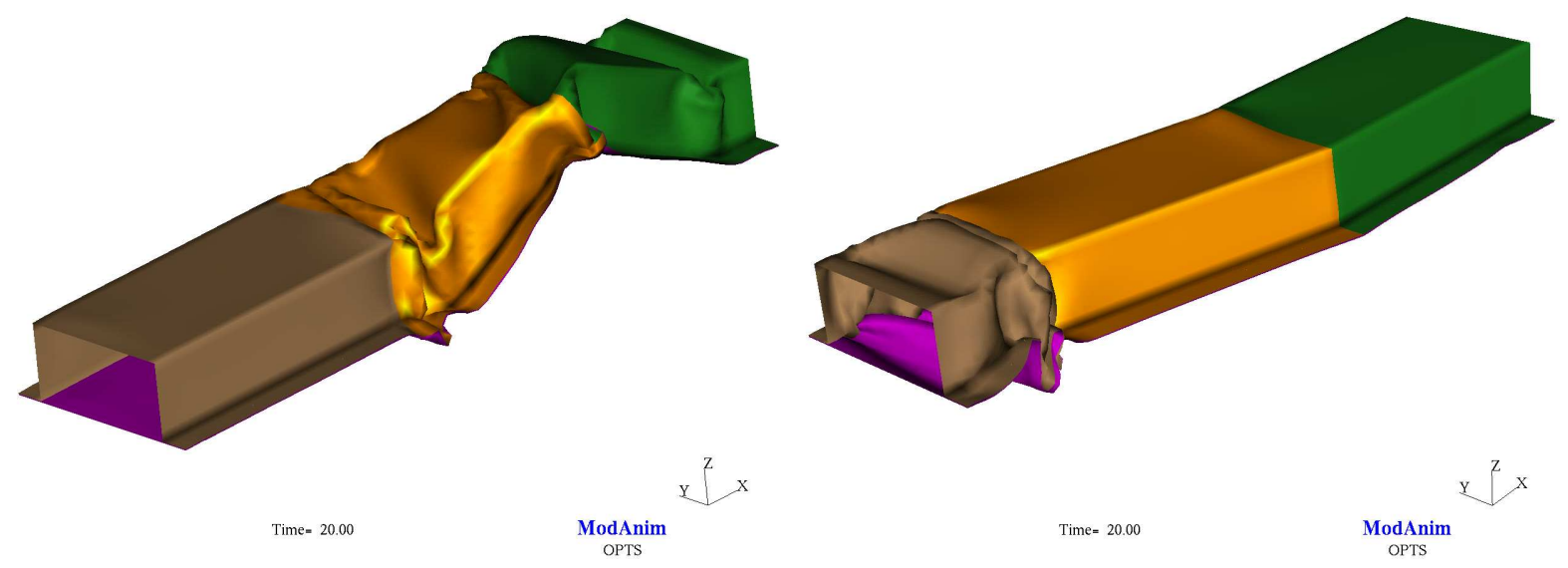

Figure 3: left: buckling type deformation, little absorbed energy; right: regular folding, good energy management

its unsatisfactory behavior.

In our problem there are 8 basic random variables. Their description and properties presented in Tab. 1. They correspond to thicknesses of the sheet metal parts, material parameters and initial conditions. To address the problem of uncertain quality of the spotweld connections $3(\approx 5 \%)$ randomly selected spring elements are always being deleted from the model, each time the crash analysis is performed. In the first part of the analysis, the MPP search, such a simplified approach may be considered as a way of accounting for some average influence of spot weld failures. Choosing randomly the spot weld elements to be deleted an additional noise effect is introduced to the LSF computations. Therefore a specially adapted method, like the one described in Sec. 3, is needed.

The crash duration is $20 \mathrm{~ms}$. In order to define the reliability analysis problem an insufficient energy absorbtion is assumed as the failure event. The minimal admissible value of absorbed energy $e_{\min }$ is taken to be equal to $6000 \mathrm{~J}$, which is about $80 \%$ of the energy absorbed by the nominal beam (corresponding to the mean values of random variables and perfect spot welds). Hence, the limit state function can be expressed as

$$
g(\boldsymbol{X}, \boldsymbol{A})=1-\frac{e_{\min }}{e(\boldsymbol{X}, \boldsymbol{A})}
$$

where $e(\boldsymbol{X}, \boldsymbol{A})$ is the energy absorbed by the beam and $\boldsymbol{A}$ stands for a vector of discrete twopoint distributed random variables accounting for the good/failed state of spot welds. It is 


\begin{tabular}{c|l|c|c|c} 
& \multicolumn{1}{|c|}{ Description } & Distribution & Mean & Std. dev. \\
\hline$X_{1}$ & $t_{1}$ - thickness of the part 1 & lognormal & $1.5[\mathrm{~mm}]$ & $0.075[\mathrm{~mm}]$ \\
\hline$X_{2}$ & $t_{2}$ - thickness of the part 2 & lognormal & $1.5[\mathrm{~mm}]$ & $0.075[\mathrm{~mm}]$ \\
\hline$X_{3}$ & $t_{3}$ - thickness of the part 3 & lognormal & $1.5[\mathrm{~mm}]$ & $0.075[\mathrm{~mm}]$ \\
\hline$X_{4}$ & $t_{4}$ - thickness of the part 4 & lognormal & $1.0[\mathrm{~mm}]$ & $0.05[\mathrm{~mm}]$ \\
\hline$X_{5}$ & $\sigma_{0}$ - yield stress & lognormal & $180[\mathrm{MPa}]$ & $15[\mathrm{MPa}]$ \\
\hline$X_{6}$ & $E$ - Young modulus & lognormal & $210000[\mathrm{MPa}]$ & $21000[\mathrm{MPa}]$ \\
\hline$X_{7}$ & $\begin{array}{l}v_{0}^{y}-y \text { component of the initial } \\
\text { velocity of impacting mass }\end{array}$ & normal & $0[\mathrm{~m} / \mathrm{s}]$ & $1.5[\mathrm{~m} / \mathrm{s}]$ \\
\hline$X_{8}$ & $\begin{array}{l}v_{0}^{z}-z \text { component of the initial } \\
\text { velocity of impacting mass }\end{array}$ & normal & $0[\mathrm{~m} / \mathrm{s}]$ & $1.5[\mathrm{~m} / \mathrm{s}]$
\end{tabular}

Table 1: Random variables of the s-rail crash problem

assumed that every realization of $\boldsymbol{A}$ produces 3 failed spot welds, which leads to removing the corresponding finite elements from the model. It was observed that due to random spot weld failures, the scatter of values of such defined LSF, measured by the maximal deviation from the sample mean, can be up to 0.07 , depending on a given realization $\boldsymbol{x}$ of the vector $\boldsymbol{X}$. Since the analytical form of the joint probability density function of $\boldsymbol{A}$ variables is unknown and it is impossible to include them in the set of basic random variables $\boldsymbol{X}$, in the first part of the algorithm, due to the strategy of spot weld removing, the influence of these variables manifests through the noisy character of LSF. The noise is handled by using the reliability analysis method presented in the current paper.

The algorithm follows closely the one described in the section 3. The first part of the algorithm is the most probable failure point search based on the adaptive response surface strategy. In computer simulated crash problems due to the nonlinear and noisy character of LSF the goal is to determine a vicinity of MPP rather than to find its "exact" location. Therefore, there is no reason for setting too strict convergence criteria since there is a high probability that they will never be fulfilled. In addition, the convergence criterion (described in point 6) should account somehow for the dimension of the problem. The criterion of the form $d<\varepsilon$, where $d$ is the distance between the last two iteration points (box centers) and, e.g., $\varepsilon=0.1$, may be too restrictive for problems with many variables. A possible modification of this criterion could be $d<k \sqrt{n \varepsilon^{2}}$, the right-hand side being $k$ times the length of the diagonal of $n$-dimensional 
hypercube, its side equal to $\varepsilon$. In the current example $n=8, \varepsilon=0.15$ and $k$ is taken equal to 1 , which gives the criterion $d<0.42$. The second criterion imposed on the mean value of LSF at MPP is defined as $h\left(\boldsymbol{u}^{*}\right)<0.1$. To avoid an extensive computational effort a limit is also set on the maximal number of iterations of the first part of the algorithm. Based on numerical experiments, it is decided to restrict the number of iterations to 15 .

Another change with respect to the original algorithm consists in a different strategy of reducing the trust region. In [23] it was proposed to reduce the size of the trust region to the half of the size from previous iteration. Again, from numerical tests we have found that such an approach can often lead to a rapid reduction of the trust region and, in consequence, undesirable behavior of the algorithm. In such cases, the response surface is based on a very localized sample of points, which, accounting for the noise influence, often leads to design point approximations outside the trust region. This effect is particularly important in high dimensional sample spaces. In the version of the algorithm implemented for this study the volume of the trust region ( $n$ dimensional hypercube) rather than its size is reduced by a constant factor. It is assumed that if the current MPP approximation is inside the trust region then the new volume is taken to be $25 \%$ of the current one. This leads to a simple formula for the size reduction

$$
b_{i}=0.25^{\frac{1}{n}} b_{i-1}
$$

where $b_{i}$ and $b_{i-1}$ are the current and the previous trust region sizes, respectively. For $n=8$ variables and initial size of the trust region equal to 6 such a strategy produces the following sequence of sizes: $6,5.05,4.24,3.57,3,2.52, \ldots$, while the halving approach would give $6,3,1.5,0.75,0.325,0.1625, \ldots$

In the analysis the liner response surface and the moving least squares strategy for weighted regression are employed. The OLH design with $4 n=32$ points is used as the plan of experiments.

The results of the first part of the algorithm are shown in Fig. 4. In the left hand side graph there is presented the history of changes of the FORM reliability index corresponding to subsequent iterations of MPP. Labels "IN" and "OUT" placed by the data point markers indicate whether MPP is found inside or outside the current trust region, respectively. The right hand side graph shows how the value of the convergence criterion changes over iterations. The criterion is 

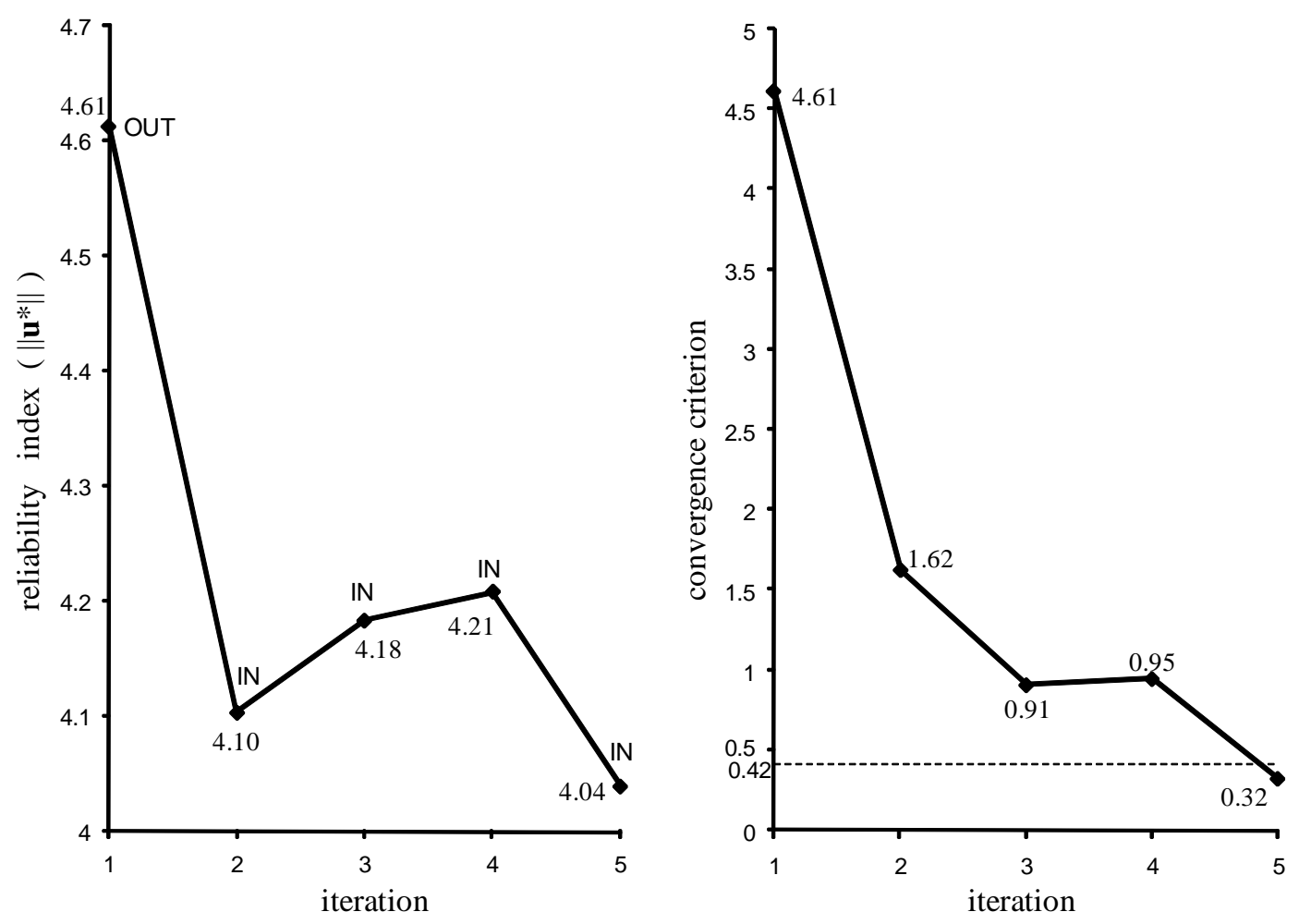

Figure 4: Design point search: reliability index changes history and the convergence (distance) criterion history

fulfilled after 5 iterations and the following results are obtained:

$$
\begin{aligned}
& \beta^{\mathrm{I}}=\beta_{\mathrm{FORM}}=4.04, \quad P_{f}^{\mathrm{I}}=2.7 \cdot 10^{-5}, \\
& \boldsymbol{u}^{*}=\{-0.89,-0.29,-1.20,-1.26,-2.19,-0.80,1.92,-1.81\}, \\
& h\left(\boldsymbol{u}^{*}\right)=0.0038
\end{aligned}
$$

No. of LSF calls $=213$,

where the superscript I stands for the first part of the algorithm. Analyzing coordinates of the point $\boldsymbol{u}^{*}$ it can be noticed that the most probable failure event will occur if thicknesses of parts 3 and 4 and the yield stress are well below their respective mean values and there is an important lateral component of the velocity of impacting mass. It should also be noticed here that the point $\boldsymbol{u}^{*}$ corresponds to the most probable realization of the variables $\boldsymbol{X}$ leading to the failure event and some averaged influence of spot weld failures ( $\boldsymbol{A}$ variables). Hence, it cannot be treated as the equivalent of the most probable failure point obtained for the problem where the random 
variables $\boldsymbol{A}$ with a known joint probability distribution would be included in the set of basic random variables. Accounting for $4 n$ sample points used to fit each linear response surface, MPP verification calls and the computation of the average LSF value at the final MPP the total number of Radioss calls in the first part of the algorithm is 213.

At the beginning of the importance sampling part of the algorithm $m_{0}=80$ new points are generated using the PDF $\varphi_{n}\left(\boldsymbol{u}, \boldsymbol{u}^{*}, \boldsymbol{I}\right)$ and the OLH design. Then, the stage of building the multi-modal sampling density, as described by points 1-4 in Sec. 3.2, is carried out with the only modification concerning the sample size. It is decided to make it equal to $5 \tilde{k}, \tilde{k}$ being the number of the most important representative points. They are chosen as points with the highest weight values satisfying the criterion that the sum of their corresponding weights is grater than 0.75. The target value for the coefficient of variation $\nu_{\widehat{P}_{f}}$ is set equal to 0.2 . The final form of multi-modal sampling density is obtained after 18 iterations (1195 Radioss calls). It is the function of the type (24) based on 17 representative points with the following weights:

$$
\begin{aligned}
& \hat{\boldsymbol{w}}=\{0.195,0.169,0.138,0.064,0.057,0.051,0.045,0.043,0.035 \text {, } \\
& 0.035,0.034,0.031,0.026,0.024,0.019,0.019,0.017\} \text {. }
\end{aligned}
$$

There are three dominating representative points that are in approximately the same distance from the origin, $\boldsymbol{u}=\mathbf{0}$, as the MPP localized in the first part of the algorithm. In Figs. 5 and 6 there are presented the sampling history graphs of the weight values and the distances from the origin corresponding to the first five representative points. The numbers shown next to the point markers of the line \#1 in Fig. 5 stand for the total number of representative points.

It can be noticed by comparing Figs. 5 and 6 with the evolution of $\widehat{P}_{f}$ in Fig. 7 that substantial changes of the probability of failure estimate between, approximately, the 500 -th and the 900 -th generated points are connected to sampling using a better adapted sampling density. In Fig. 8 there is shown the history of changes of the coefficient of variation $\nu_{\widehat{P}_{f}}$. One may observe that the trend is not monotonic. The jump may be due to rare events of generating points with a high ratio $\varphi_{n}\left(\boldsymbol{v}_{i}, \mathbf{0}, \boldsymbol{I}\right) / s_{\boldsymbol{V}}\left(\boldsymbol{v}_{i}\right)$, see Eq. (21). The final approximation of the probability of failure and the corresponding generalized reliability index are

$$
\begin{aligned}
& P_{f}^{\mathrm{II}}=5.37 \cdot 10^{-5}, \quad \beta^{\mathrm{II}}=-\Phi^{-1}\left(P_{f}^{\mathrm{II}}\right)=3.87, \\
& \text { No. of LSF calls }=1195,
\end{aligned}
$$

where the superscript II is for the second part of the algorithm. 


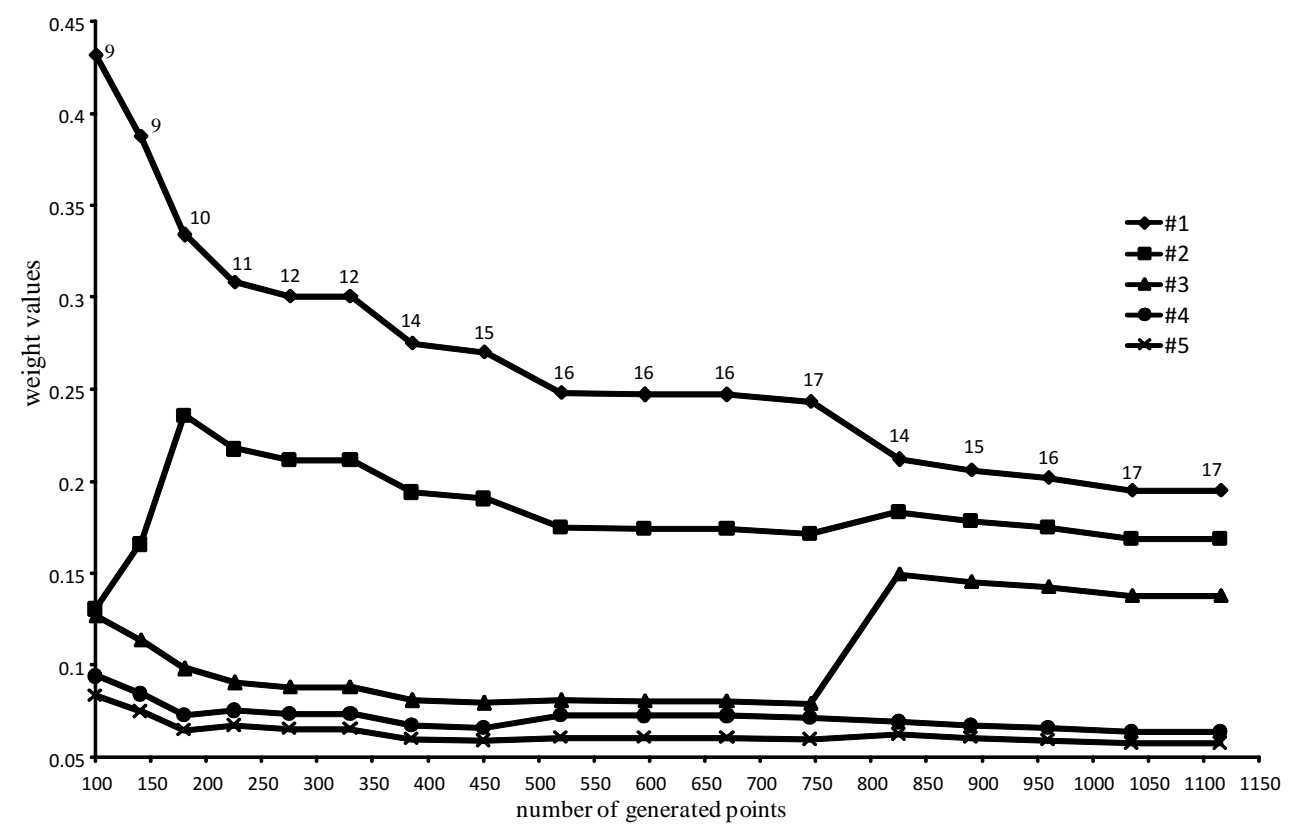

Figure 5: Multi-modal importance sampling: Changes of weight values for the first five representative points. Numbers above the line \#1 denote the total number of representative points.

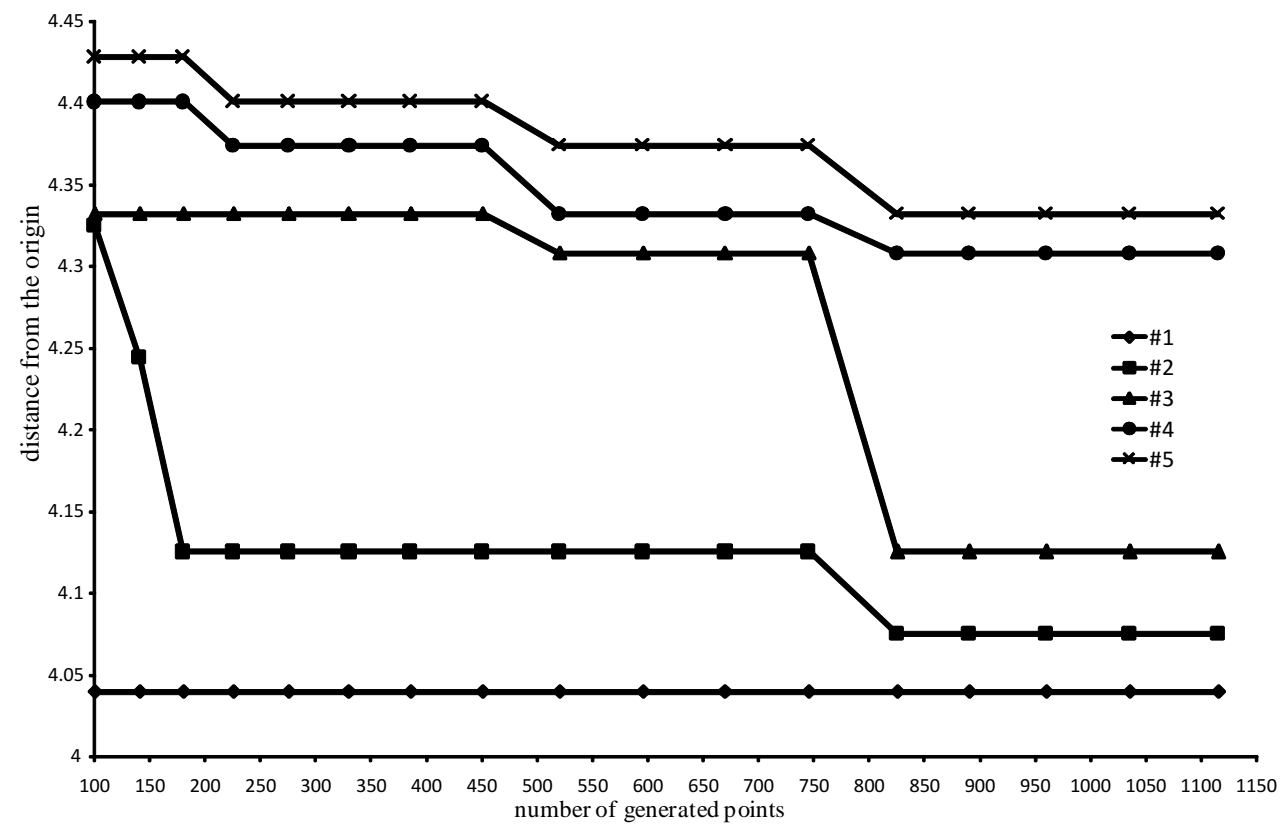

Figure 6: Multi-modal importance sampling: Distances from the origin $\boldsymbol{u}=\mathbf{0}$ of the first five representative points. 


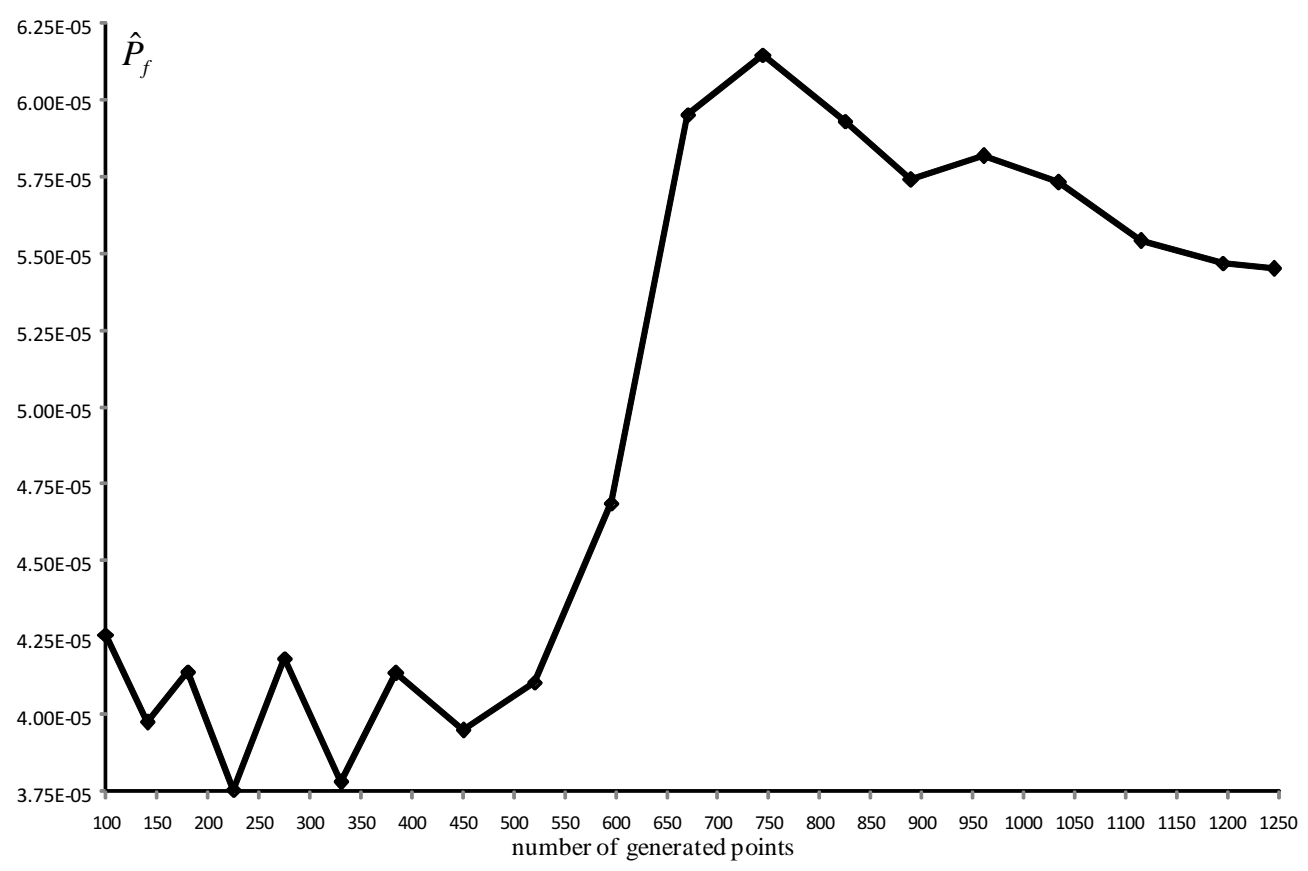

Figure 7: Multi-modal importance sampling: Evolution of $P_{f}$ estimate

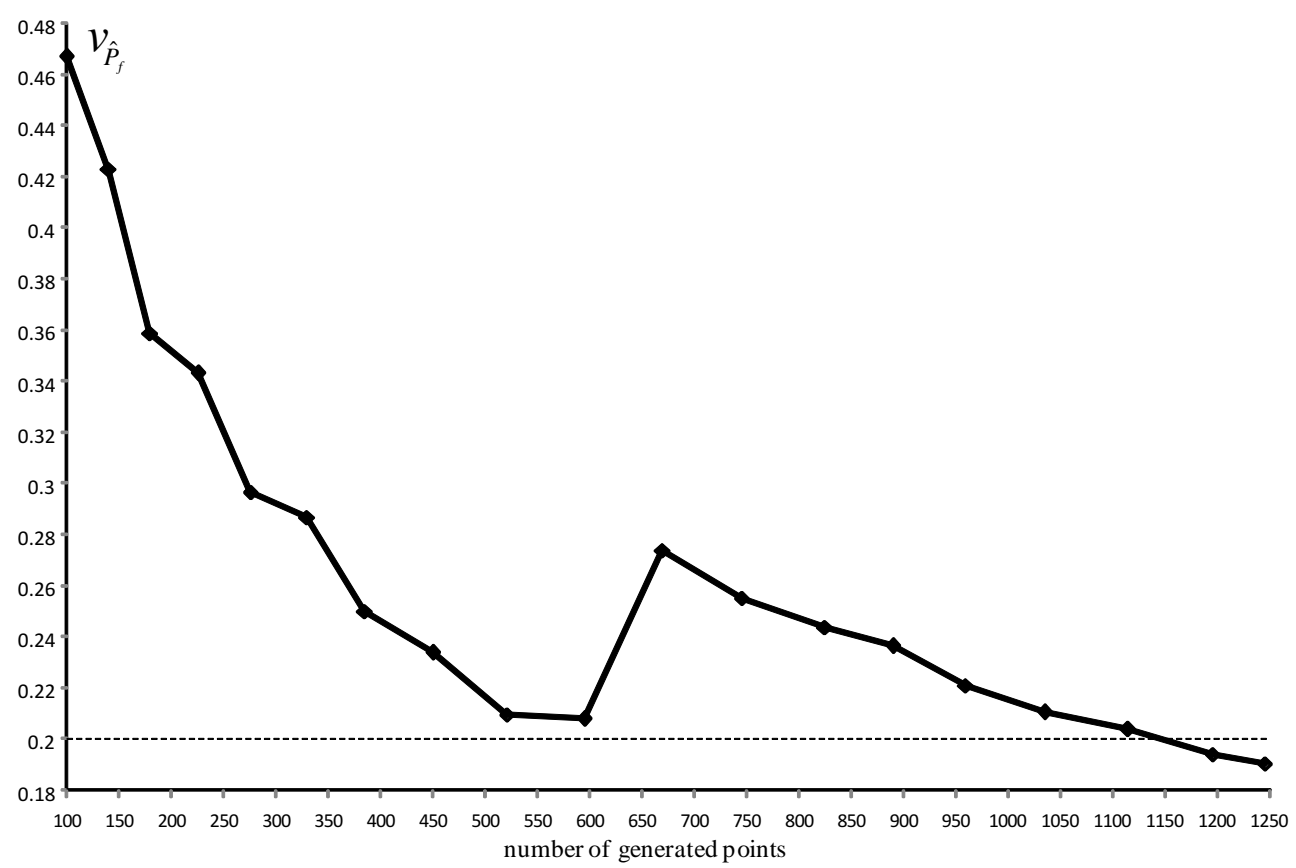

Figure 8: Multi-modal importance sampling: Evolution of the coefficient of variation of $\widehat{P}_{f}$ 
Comparing values of $P_{f}^{\mathrm{I}}$ and $P_{f}^{\mathrm{II}}$ one may conclude that the limit state function is not strongly nonlinear and it is arguable if the importance sampling correction brings much with respect to FORM approximation based on the MPP determined in the first phase of the algorithm. Even though the sampling results with two times higher failure probability than $P_{f}^{\mathrm{I}}$ it is not a qualitative change and this can be questioned whether the big computational effort is worth the effects it produced. This, however, cannot be known beforehand and in order to gain some confidence in the FORM results importance sampling serves as a method for verification.

This is interesting to assess the influence of spot weld defects on the crashworthiness reliability of the s-rail. For this purpose the analysis is repeated, applying the same algorithm but, this time all the spot welds are assumed to be in perfect shape. Below there are given the results for the first and the second part of the method, respectively.

First part, no. of LSF calls $=199$,

$$
\begin{aligned}
& \beta^{\mathrm{I}}=\beta_{\mathrm{FORM}}=4.02, \quad P_{f}^{\mathrm{I}}=2.9 \cdot 10^{-5}, \\
& \boldsymbol{u}^{*}=\{-1.14,0.15,-0.99,-1.84,-1.61,-1.30,1.81,-1.72\}, \\
& h\left(\boldsymbol{u}^{*}\right)=-0.0016,
\end{aligned}
$$

Second part, no. of LSF calls $=660$,

$$
P_{f}^{\mathrm{II}}=1.9 \cdot 10^{-5}, \quad \beta^{\mathrm{II}}=-\Phi^{-1}\left(P_{f}^{\mathrm{II}}\right)=4.12 .
$$

From the comparison of failure probabilities corresponding to the cases with and without spot weld failures (equations (30) and (33), respectively) it can be immediately concluded that the spot weld quality has an important influence on the s-rail reliability. It is about 3 times less probable that the component with undamaged spot weld joins fails to absorb the required amount of impact energy than the component with some spot weld defects. The most probable failure points (32) and (29) are different, however, they are qualitatively very similar. They reflect an intuitive failure scenario with the weaker material, thinner metal sheets and significantly non-axial impact direction.

\section{Conclusions}

Deformation behavior of thin-walled steel or aluminium components in dynamic crash is very often bifurcation driven, therefore, random spot weld connection failures may determine failure modes of the entire component. In the paper there is proposed a method of accounting for 
spot weld failures in reliability analysis of crash related problems. Due to a great number of such joins, a precise modelling of spot weld parameters is rather unrealistic and would lead to unmanageable stochastic model. The suggested approach, based on introducing an additional uncertainty (random noise) to computed limit state function values, requires a specialized algorithm employing a response surface approximation of the limit state function and adaptive sampling techniques.

The algorithm investigated in the current paper, on test examples as well as on the s-rail crash problem is an improved version of the method proposed by Zou et al. in [23]. The presented approach seems to be well suited for the crashworthiness reliability analysis applications. However, its performance depends on many arbitrarily selected parameters. They should be carefully chosen to reduce the computational cost and not to impair the accuracy of the method for a broad class of crashworthiness reliability problems. Such a study has been done and some guidelines are formulated, nevertheless, this is still a challenging task for further research.

The performed s-rail reliability analysis example has shown that the effect of spot weld failures on the crashworthiness reliability of the component is not negligible and should be accounted for in realistic analysis.

\section{Acknowledgments}

The principal part of the presented work was realized under the framework of the EC-FP6 APROSYS Integrated Project. The partial support from the Polish Committee for Scientific Research grant, No. N519 010 31/1601 is also gratefully acknowledged. The authors would like to express their gratitude to the company Altair Development France for granting them the license for the Radioss software. Prof. K. Dolinski from the Institute of Fundamental Technological Research of Polish Academy of Sciences is thanked for his helpful comments.

\section{References}

[1] T. Abdo and R. Rackwitz. A new beta-point algorithm for large time-invariant and timevariant reliability problems. In A. Der Kiureghian and P. Thoft-Christensen, editors, Reliability and Optimization of Structural Systems '90, Proc. 3rd WG 7.5 IFIP Conf., Berkeley, 26-28 March 1990, pages 1-12, Berlin, 1991. 
[2] K. Doliński. First-order second-moment approximation in reliability of structural systems: critical review and alternative approach. Structural Safety, 1:211-231, 1983.

[3] S. Donders, M. Brughmans, L. Hermans, C. Liefooghe, and W. Van der Auweraer, H. Desmet. The robustness of dynamic vehicle performance to spot weld failures. $F i$ nite Elements in Analysis and Design, 42:670-682, 2006.

[4] E.N. Dvorkin and K.J. Bathe. A continuum mechanics based four-node shell element for general nonlinear analysis. Eng Comput, 1:77-88, 1984.

[5] L. Gu, R.J. Yang, C.H. Tho, M. Makowski, O. Faruque, and Y. Li. Optimization and robustness for crashworthiness of side impact. International Journal of Vehicle Design, 26:348-360, 2001.

[6] I. Hagiwara and Q. Z. Shi. Vehicle crashworthiness design using a most probable optimal design method. In K.J. Bathe, editor, First MIT Conference on Computational Fluid and Solid Mechanics, Boston, pages 637-640. Elsevier Science Ltd., 2001.

[7] G. Johnson and W. Cook. A constitutive model and data for metals subjected to large stains, high strain rates and high temperatures. In 7th Symposium on Ballistics, The Hague, 1983.

[8] A. Karamchandani, P. Bjerager, and C.A. Cornell. Adaptive importance sampling. In A.H.-S. Ang, M. Shinozuka, and G.I. Schuëller, editors, Proceedings 5th International Conference on Structural Safety and Reliability, San Francisco. ASCE, 1989.

[9] H. Kurtaran, A. Eskandarian, D. Marzougui, and N.E. Bedewi. Crashworthiness design optimization using successive response surface approximations. Computational Mechanics, 29:409-421, 2002.

[10] M. Liefvendahl and R. Stocki. A study on algorithms for optimization of Latin hypercubes. Journal of Statistical Planning and Inference, 136:3231-3247, 2006.

[11] P.-L. Liu and A. Der Kiureghian. Optimization algorithms for structural reliability. Structural Safety, 9:161-177, 1991.

[12] H.O. Madsen, S. Krenk, and Lind N.C. Methods of Structural Safety. Prentice-Hall, 1986. 
[13] Mecalog SARL, 2 Rue de la Renaissance 92160 Antony, France. RADIOSS Input Manual, Version 4.2, 2000.

[14] R.E. Melchers. Simulation in time-invariant and time-variant reliability problems. In R. Rackwitz and P. Thoft-Christensen, editors, Reliability and Optimization of Structural Systems '91, Proc. 4th IFIP WG 7.5 Conf., Munich, 11-13 September 1991, pages 39-82. Springer-Verlag, 1992.

[15] R.E. Melchers. Structural Reliability Analysis and Predictions, 2nd Ed. Wiley, 1999.

[16] M. Redhe, J. Forsberg, T. Jansson, P.-O. Marklund, and L. Nilsson. Using the response surface methodology nad the d-optimality criterion in crashworthiness related problems. Struct. Multidisc. Optim, 24:185-194, 2002.

[17] K. Schittkowski. User's guide for the nonlinear programming code nlpql. Handbook to optimization program package NLPQL, University of Stuttgart - Institute for Informatics, Germany, 1985.

[18] U. Schramm. Multi-disciplinary optimization for nvh and crashworthiness. In K.J. Bathe, editor, First MIT Conference on Computational Fluid and Solid Mechanics, Boston, pages 721-724. Elsevier Science Ltd., 2001.

[19] G.I. Schuëller and R. Stix. A critical appraisal of methods to determine failure probabilities. Structural Safety, 4:293-309, 1987.

[20] N. Stander, W. Roux, M. Giger, M. Redhe, N. Fedorova, and J. Haarhoff. Crashworthiness optimization in LS-OPT: case studies in metamodelling and random search techniques. In Proc. 4th European LS-DYNA Users Conference, Ulm, 22-23 May, 2003.

[21] R. J. Yang, L. Gu, C. Soto, G. Li, and T. Tyan. Developments and applications of structural optimization and robustness methods in vehicle impacts. In ASME International Mechanical Engineering Congress, Proceedings of IMECE2004, November 13-19. Anaheim, California, 2004.

[22] R. J. Yang, C. H. Tho, C. C. Wu, D. Johnson, and J. Cheng. A numerical study of crash optimization. In ASME Design Engineering Technical Conference, Proceedings of DETC 99, September 12-15. Las Vegas, Nevada, 1999. 
[23] T. Zou, S. Mahadevan, Z.P. Mourelatos, and P. Meernik. Reliability analysis of automotive body-door subsystem. Reliability Engineering and System Safety, 78(3):315-324, 2002.

[24] T. Zou, S. Mahadevan, Z.P. Mourelatos, and P. Meernik. Reliability analysis of systems with nonlinear limit states; application to automotive door closing effort. In Reliability \& Robust Design in Automotive Engineering, pages 19-29. SAE International, 2003. 